\title{
Analysis and Design of a Cylindrical EBG-Based Directive Antenna
}

\author{
Halim Boutayeb, Member, IEEE, Tayeb A. Denidni, Senior Member, IEEE, Kouroch Mahdjoubi, Member, IEEE, \\ Anne-Claude Tarot, Member, IEEE, Abdel-Razik Sebak, Senior Member, IEEE, and Larbi Talbi, Senior Member, IEEE
}

\begin{abstract}
In this paper, a cylindrical electromagnetic bandgap (CEBG) structure composed of infinite metallic wires is analyzed, designed and used as a model to develop a new reconfigurable directive antenna. This structure is circularly and radially periodic, and it is excited at its center using an omnidirectional source. The analysis is based on calculating the transmission and reflection coefficients of a single cylindrical frequency selective surface (FSS) and then, considering only the fundamental mode interaction, deducing the frequency response of the CEBG structure composed of multiple cylindrical FSSs. For this structure, new analytical formulas are derived, and their accuracy is assessed compared to those obtained by the finite-difference time-domain method. As in rectangularly periodic structure case, the frequency response of the CEBG structure exhibits pass-bands and bandgaps, and it is possible to obtain directive beams by introducing defects in the periodic structure. Using this concept, a new antenna was developed to obtain a controllable directive beam. An antenna prototype, without control, was designed, fabricated, and tested. An excellent agreement was obtained between theory and experiment for both return loss and radiation patterns.
\end{abstract}

Index Terms-Cylindrical structures, directive antennas, electromagnetic bandgap (EBG) materials, periodic structures.

\section{INTRODUCTION}

$\mathbf{R}$ ECENTLY, photonic bandgap (PBG) materials [1], [2] have drawn significant attention in physics and engineering due to their analogy to semiconductor crystals, where electron bandgaps can be found. In microwave and antenna domains, they are known as electromagnetic bandgap (EBG) structures. EBG materials are periodic structures characterized by the forbidden propagation of electromagnetic waves whose frequencies belong to frequency bandgaps, and also characterized by the ability to open localized electromagnetic modes inside the forbidden frequency bandgap by introducing defects into the periodical structures. EBG structures, rectangularly periodic, have been used in several applications, such as suppressing surfaces waves [3], designing directive antennas with a single feed [4], [5], and creating a controllable beam [6], [7]. In this paper, a new concept of circularly and radially

Manuscript received March 19, 2005; revised July 12, 2005.

H. Boutayeb and T. A. Denidni are with the INRS-EMT, University of Quebec, Montreal, QC H5A 1K6, Canada (e-mail: boutayeb@emt.inrs.ca).

K. Mahdjoubi and A.-C. Tarot are with the Institut d'Électronique et de Télécommunications de Rennes (IETR ), UMR CNRS 6164, 35042 Rennes, France.

A.-R. Sebak is with the Electrical and Computer Engineering Department, Concordia University, Montreal, QC H3G 1M8, Canada.

L. Talbi is with the Propagation and Satellite (PSAT) Labs of Communications Research Center (CRC), Ottawa, Canada, on leave from the Université du Québec en Outaouais, Gatineau, QC J8X 3X7, Canada.

Digital Object Identifier 10.1109/TAP.2005.861560 periodic structures, i.e., cylindrical EBG (CEBG) structures, is investigated in order to demonstrate the advantage of their circular symmetry, which can allow a reconfigurable directive beam over $360^{\circ}$ range.

It is known that the transmission and reflection characteristics of periodic structures, composed of multiple layers of planar frequency selective surfaces (FSSs), can be obtained using the cascading scattering matrix method [8]. This method is inspired from that of cascading networks in the circuit theory, and it reduces considerably computing time compared to the conventional ones that calculate the overall scattering from the structure. The cascading approach is well suited to the design process because it is possible to evaluate the effect of any changing in one layer or in the period without repeating the entire computing process. The same approach may be applied to CEBG structures by calculating the transmission and reflection characteristics of a single cylindrical FSS. Cylindrical FSSs have been studied by several investigators [9]-[12]. One of the principal objectives was to calculate scattered fields of cylindrical surface using Floquet harmonics. In [9], both internal line source excitation and plane wave excitation were considered. In [10], a numerical technique based on the Conjugate Gradient with Fast Fourier Transform was used. In [11], [12], the cylindrical FSS has been used as a waveguide, and the propagation constant has been studied. However, from these contributions, the transmission and reflection coefficients of the cylindrical FSS have not been calculated yet. The challenge in this structure is the existing of multiple reflections between the cylindrical FSS and its center.

This paper presents the design of a new reconfigurable directive antenna using a simple but efficient model based on CEBG structures and a cascading approach. The problem of the characterization of a single cylindrical FSS is resolved and the multiple reflections of cylindrical waves between cylindrical FSSs are developed for the first time. In this work, the periodic structures are composed of infinite metallic wires and only the fundamental mode interaction between cylindrical layers is considered. The proposed approach is well suited for the design process of the proposed antenna. An equivalent network approach for circular cylindrical FSSs which considers multiple modes interactions can be found in [13], but it is more time consuming that the present approach. Using the CEBG structure model, an application for creating a controllable beam over $360^{\circ}$ range is proposed. The concept of controllable beam with reconfigurable EBG structures has been proposed in [6], [7], where the authors have proposed to commute between continuous and discontinuous wire structures for reconfiguring 


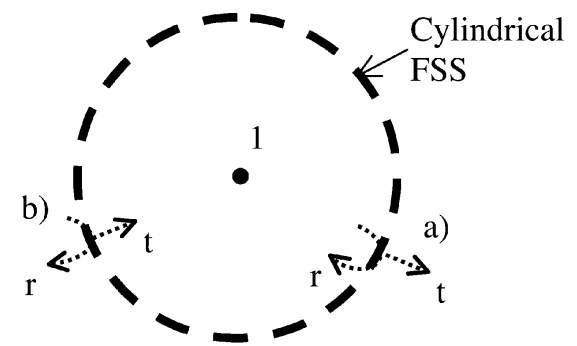

Fig. 1. Characteristics of a cylindrical FSS: (a) reflection and transmission coefficients for a cylindrical wave coming from inside to outside, (b) reflection and transmission coefficients for a cylindrical wave coming from outside to inside.

the radiation patterns of an antenna. Indeed, these two structures behave differently at low frequencies, by presenting a pass-band and a stop-band for discontinuous and continuous wires cases, respectively. To obtain the commutation, the authors have placed periodically diodes in the broken wires. When the diodes are switched on, the structure behaves as a continuous wire medium, if the impedance of the diodes are neglected, whereas when the diodes are switched off, the structure behaves as a discontinuous wire medium. We have recently presented an analysis of the band structure of the discontinuous wire medium for different wire diameters and lengths in [14]. In [6], [7], EBG structures with rectangular mesh have been used. However, EBG structures with rectangular mesh present a major drawback: they do not make possible to obtain a beam turning on $360^{\circ}$ with any angle of direction and any step. Cylindrical EBG structures allow to overcome this drawback.

In the next section, new formulas to extract the transmission and reflection coefficients of a single cylindrical FSS are outlined, completing our previous work [15]. Then, in Section III, we develop an iterative formulation to handle multilayered CEBG structures. The approach is validated using finite-difference time-domain (FDTD) method. As an application, a new reconfigurable directive antenna, based on CEBG structures with defects, is described in Section IV. Finally, in Section V, an antenna prototype is designed, fabricated and tested. The fabricated antenna does not have a control yet in order to test first the directivity characteristic of CEBG structures with defects. Both theoretical and experimental results are presented and discussed, which demonstrate the accuracy and usefulness of the present approach.

\section{Characterization of a Single Cylindrical FSS}

In this section, the transmission and reflection coefficients of a single cylindrical FSS are calculated. These coefficients can not be directly obtained as in the planar case because of the multiple reflections between the cylindrical FSS and its center. It is clear that, for a current line source or no object in the center, the reflection coefficient at the center is equal to 1 . Now, let us consider the transmission $(t)$ and reflection $(r)$ coefficients of the cylindrical FSS as shown in Fig. 1. Using the principle of reciprocity, these coefficients are the same whatever the structure is illuminated by a cylindrical wave coming from inside or from outside. To study these coefficients, we consider an incident cylindrical wave coming from the center as shown in Fig. 2.

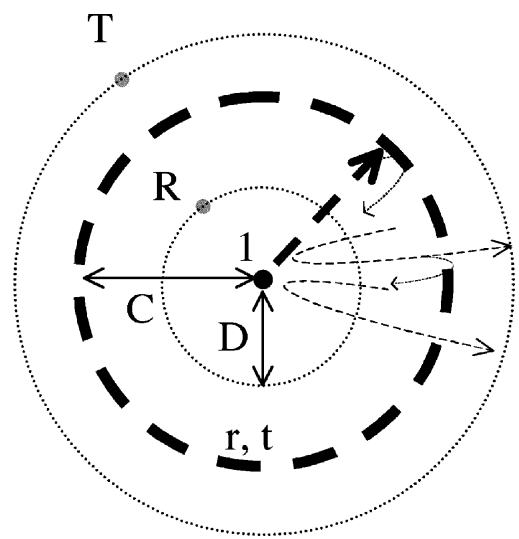

Fig. 2. Cylindrical FSS illuminated by a cylindrical wave coming from the center. Multiple reflections between the center and the surface lead to the coefficients $T$ and $R$.

Calculating the normalized fields at points outside and inside the cylindrical cavity leads to the coefficients $T$ and $R$ as illustrated in Fig. 2. In Section II-A, analytical relations between $(t, r)$ and $(T, R)$ coefficients are developed, and in Section II-B, the coefficients $T$ and $R$ are calculated for the particular case of a single surface composed of metallic wires.

\section{A. Extraction of the Reflection and Transmission Coefficients}

Using the multiple reflections between the cylindrical FSS and its center as shown in Fig. 2, the coefficient $T$ can be expressed as a function of $t$ and $r$

$$
\begin{aligned}
T & =t \sum_{n=0}^{\infty} r^{n} e^{-j n 2 \eta_{0}(k C)} \\
& =\frac{t}{1-r e^{-j 2 \eta_{0}(k C)}}
\end{aligned}
$$

where $k$ is the free space wave number, $C$ is the radius of the cylinder and $\eta_{0}(x)$ is the phase of the cylindrical wave

$$
\eta_{0}(x)=\arctan \left(\frac{N_{0}(x)}{J_{0}(x)}\right)
$$

where $J_{0}(x)$ and $N_{0}(x)$ are the first and second kind Bessel functions of zero order.

To obtain $R$, an observation point at the distance $D(D<C)$ from the center is considered. Referring to Fig. $2, R$ can be expressed as a function of $t$ and $r$

$$
R=\frac{1+r e^{-j 2 \eta_{0}(k C)+j 2 \eta_{0}(k D)}}{1-r e^{-j 2 \eta_{0}(k C)}} .
$$

From (1) and (3), one can show that $t$ and $r$ can be expressed as

$$
\begin{aligned}
t & =\frac{1+e^{j 2 \eta_{0}(k D)}}{R+e^{j 2 \eta_{0}(k D)}} T \\
r & =\frac{R-1}{R+e^{j 2 \eta_{0}(k D)}} e^{j 2 \eta_{0}(k C)}
\end{aligned}
$$




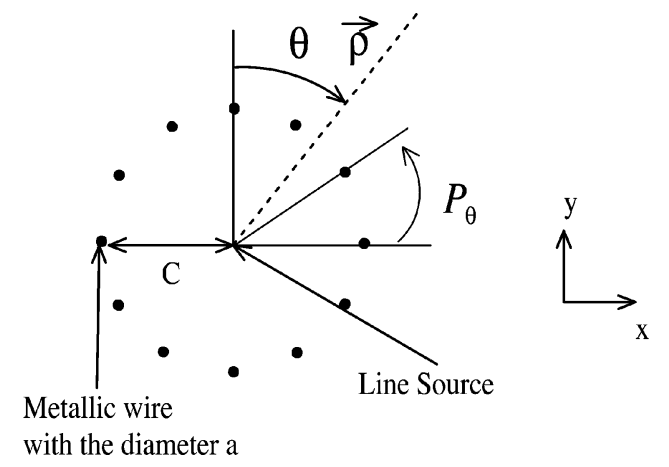

Fig. 3. Periodic cylindrical structure made of infinite long metallic wires and illuminated by a TM cylindrical wave coming from the center.

\section{B. Calculation of the Scattered Field}

In this section, we present a simple method to calculate $T$ and $R$ coefficients for a single cylindrical FSS composed of infinite long metallic wires as illustrated in Fig. 3. The parameters of this structure are the diameter of the wires $a$, the radius $C$, and the angular period $P_{\theta}$. We also denote by $P_{t}\left(P_{t}=P_{\theta} C\right)$ the transversal period and by $N\left(N=360 / P_{\theta}\right)$ the number of wires.

The structure is illuminated by an infinite line source, with a TM cylindrical wave. The transverse electric field $E(\vec{\rho})$ is a function of the frequency and the position $\vec{\rho}$ from the center. According to [16], this transverse field is equal to the incident field plus the field scattered by the wires. Thus, $E(\vec{\rho})$ can be written as

$$
E(\vec{\rho})=E_{\mathrm{inc}}(\vec{\rho})+\sum_{n=0}^{N-1} K_{n} E_{s n}(\vec{\rho})
$$

where $E_{\text {inc }}(\vec{\rho})=H_{0}^{2}(k\|\vec{\rho}\|), H_{0}^{2}$ is the Hankel function of the second kind and of zero order, and $E_{s n}$ is the scattered field by the wire $n(n=0, \ldots, N-1) . K_{n}$ are unknown coefficients due to the interactions between the wires. Because of the symmetry of the structure, the coefficients $K_{n}$ are all identical, i.e., $K_{n}=K$, and we can write

$$
\sum_{n=0}^{N-1} K_{n} E_{s n}(\vec{\rho})=K \sum_{n=0}^{N-1} E_{s n}(\vec{\rho})
$$

For a small wire diameter $a$, the scattered fields $E_{s n}$ can be written in terms of the Hankel function $H_{0}^{2}$ as

$$
\begin{aligned}
E_{s n}(\vec{\rho}) & =H_{0}^{2}\left(k \sqrt{C^{2}+\|\vec{\rho}\|^{2}-2 C\|\vec{\rho}\| \cos \left(\frac{n 2 \pi}{N}-\theta\right)}\right) \\
n & =0, \ldots, N-1 .
\end{aligned}
$$

$K$ is obtained by imposing $E\left(\overrightarrow{\rho_{s}}\right)=0$ on the wire surface, for the case of wire $n=0: \overrightarrow{\rho_{s}}=C \vec{y}+\overrightarrow{d s}$, where $\overrightarrow{d s}$ is the vector defined by the center of the wire $n=0$ and a point on the surface of this wire.

The equation $E\left(\overrightarrow{\rho_{s}}\right)=0$ must be satisfied at all points of the surface of the wire $n=0$. As a good approximation for a small wire diameter, $\overrightarrow{d s}$ is neglected in the expressions of
$E_{\text {inc }}\left(\overrightarrow{\rho_{s}}\right)$ and $E_{s n}\left(\overrightarrow{\rho_{s}}\right)(n=1 \ldots N-1)$. Since $E_{s 0}\left(\overrightarrow{\rho_{s}}\right)$ does not depend on the angle defined by the vector $\overrightarrow{d s}$, then the point $C \vec{y}+a / 2 \vec{y}$ can be included in the $E_{s 0}$ expression. Finally, $K$ is obtained by the following relation:

$$
K=-\frac{E_{\mathrm{inc}}(C \vec{y})}{E_{s 0}\left(\left(C+\frac{a}{2}\right) \vec{y}\right)+\sum_{n=1}^{N-1} E_{s n}(C \vec{y})} .
$$

The variable $T$ is defined as the radiated transverse electric field outside the cavity normalized by the radiated field of the source with the cylindrical surface not present, and $R$ is the transverse electric field inside the cavity at the distance $D(D<C)$ from the center normalized by the radiated field of the source with the cylindrical surface not present, then we can write

$$
\begin{aligned}
& T=\frac{E(\vec{\rho})}{E_{\text {inc }}(\vec{\rho})}, \quad\|\vec{\rho}\|>C+P_{t} \\
& R=\frac{E(\vec{\rho})}{E_{\text {inc }}(\vec{\rho})}, \quad\|\vec{\rho}\|=D<C-P_{t} .
\end{aligned}
$$

Here we can note that, after numerical studies, a minimum distance of approximately $P_{t}$, the transversal period, must be respected between the observation point and the surface to avoid non propagating waves. In this case, it can be seen that the coefficient $|T|$ does not depend on the position of the observation point as long as it is far enough from the surface, i.e., $\|\vec{\rho}\|>C+P_{t}$.

To validate the accuracy of the above expressions, the structure shown in Fig. 3 has been simulated using the FDTD method. The source is an infinite line current source and the wires are modeled using Holland et al. thin wire formalism [17]. The $T$ and $R$ coefficients are obtained with the FDTD method by carrying out two simulations: one with the structure and another without the structure for the normalization. Numerical results based on this current analysis and the FDTD simulations for $|T|$ are plotted in Fig. 4. A good agreement is observed between the results obtained by the current method and those computed by the FDTD method. The reason of a strength of $|T|$ greater than one is that the cavity modifies the matching of the line source, which is not initially matched to free space, and then the power supplied outside the cavity, at resonance, is greater than the power supplied by the line source alone. However, according to the energy conservation principle, the strengths of $|t|$ and $|r|$ are always smaller than one, as it is shown in the next section.

\section{Cylindrical FSS}

The extraction of the reflection and transmission coefficients for different cylindrical FSSs allows an interesting analysis presented in this section. For instance, we consider the following case: $C=45 \mathrm{~mm}, P_{\theta}=30^{\circ}$ and $a=1.5 \mathrm{~mm}$. The transversal period is $P_{t}=\pi / 6 \times 45 \mathrm{~mm}$. Fig. 5 shows $|t|$ and $|r|$ coefficients obtained with the proposed extraction method [using (4) and (5)].

The characteristics $|t|$ and $|r|$ of a second structure with the parameters $C=90 \mathrm{~mm}, P_{\theta}=15^{\circ}$ and $a=1.5 \mathrm{~mm}$ were computed. This structure has the same transversal period as the 


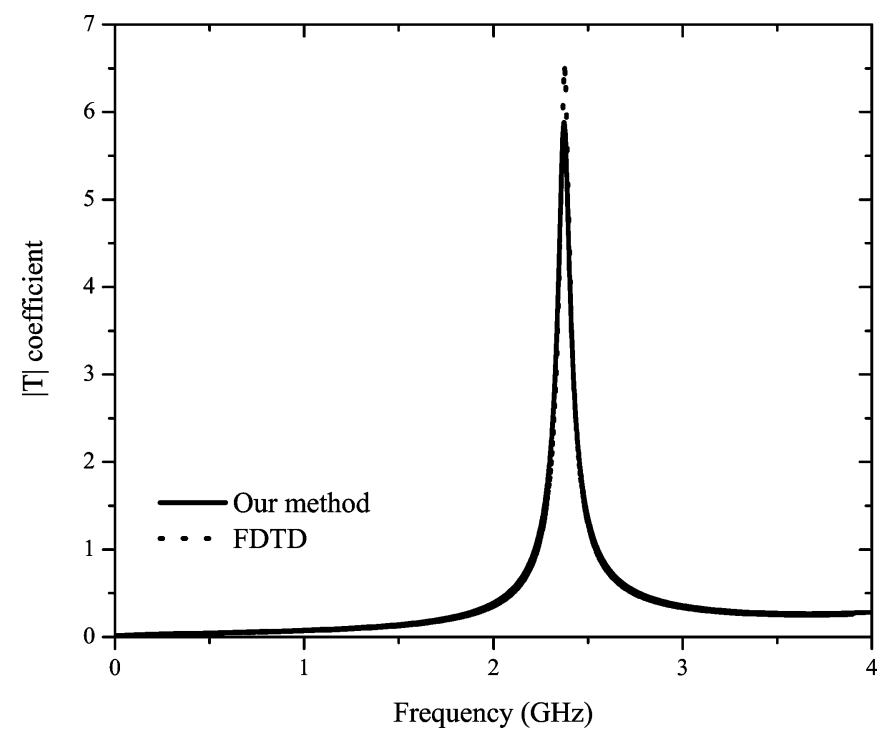

Fig. 4. Transmission coefficient $|T|$ of a single cylindrical FSS ( $a=1.5 \mathrm{~mm}$, $\left.P_{\theta}=30^{\circ}, C=45 \mathrm{~mm}\right)$.

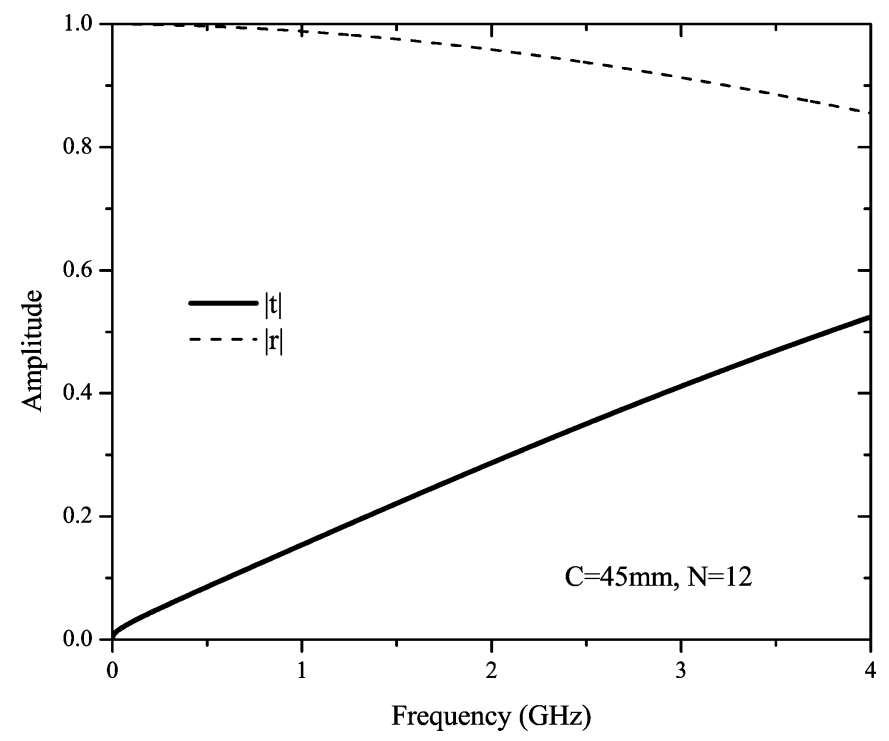

Fig. 5. Coefficients $|t|$ and $|r|$ of a single cylindrical FSS $(a=1.5 \mathrm{~mm}$, $\left.P_{\theta}=30^{\circ}, C=45 \mathrm{~mm}\right)$.

previous structure $\left(P_{t}=\pi / 3 \times 90 \mathrm{~mm}\right)$. Fig. 6 shows the coefficients $|t|$ and $|r|$ of this case. We can observe that the coefficients $|t|$ and $|r|$ are similar to the previous ones shown in Fig. 5, and the phases of $t$ and $r$ (not shown here) are also similar to the previous ones. This observation can be verified with other structures, and it can be conclude that when the transversal period is constant, the structure has the same $t$ and $r$ coefficients. EBG structures composed of planar FSSs use usually surfaces with the same characteristics. Therefore, it is possible to apply the

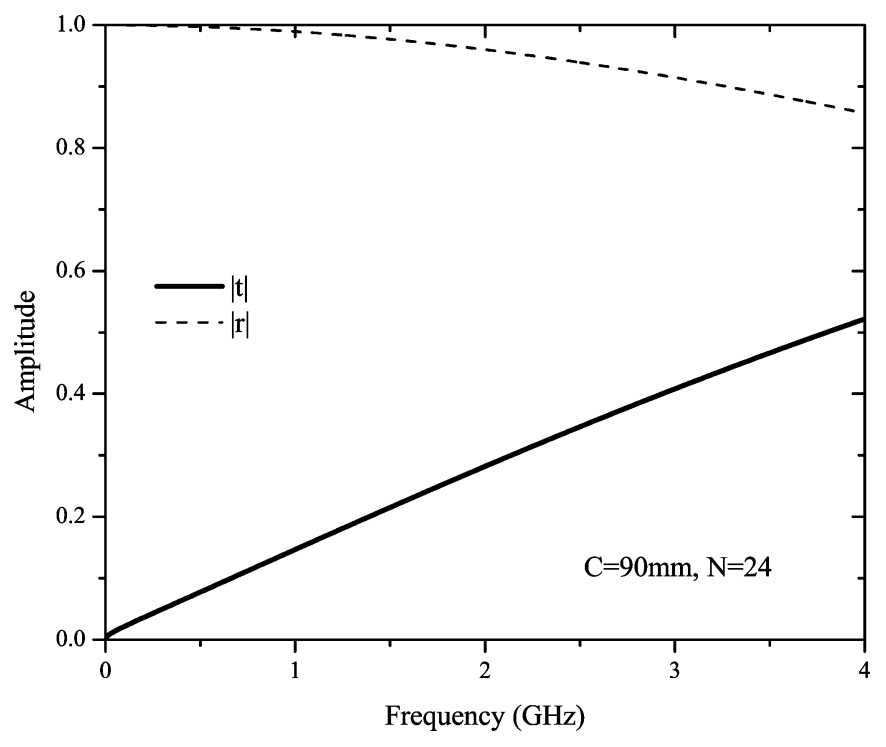

Fig. 6. Coefficients $|t|$ and $|r|$ of a single cylindrical FSS ( $a=1.5 \mathrm{~mm}$, $\left.P_{\theta}=15^{\circ}, C=90 \mathrm{~mm}\right)$.

same design for CEBG structures using cylindrical FSSs with the same transversal period.

\section{Multilayered CYLindRICAL STRUCTURES}

In this section, a new analytical iterative formulation is developed to handle cylindrical multilayered structures. A CEBG structure composed of multiple layers of cylindrical FSSs of metallic wires as shown in Fig. 7 is considered. The cylindrical surfaces are periodically spaced with the period $P_{r}$ and have the same transversal period $P_{t}$, and accordingly the same transmission and reflection coefficients. The transversal period is kept constant by modifying adequately the angular period of each layer $\left(P_{\theta 1}, P_{\theta 2}, \ldots\right)$.

Using multiple reflections and considering single-mode interactions between layers, one can show that the transmission and reflection coefficients of the CEBG structure composed of $n$ layers of cylindrical FSSs can be written as in (12) and (13), shown at the bottom of page. The transmission $\left(t_{n}\right)$ and reflection $\left(r_{n}\right)$ coefficients of a multilayered structures composed of $n$ layers of cylindrical FSSs are functions of the coefficients of the structure composed of $n-1$ layers. To our knowledge, this is the first time that analytical relations of the multiple reflections of cylindrical waves in radially periodic structures are presented. These relations are an extension to the cylindrical case of formulas for multi-sections transmission lines [18]. Taking into account the multiple reflections with the center, we obtain the coefficient $T_{n}$

$$
T_{n}=\frac{t_{n}}{1-r_{n} e^{-j 2 \eta_{0}(k C)}} .
$$

$$
\begin{aligned}
t_{n} & =\frac{t_{n-1} t}{1-r_{n-1} r e^{-j 2 \eta_{0}\left(k\left(C+(n-1) P_{r}\right)\right)+j 2 \eta_{0}\left(k\left(C+(n-2) P_{r}\right)\right)}} \\
r_{n} & =r_{n-1}+\frac{t_{n-1}^{2} r e^{-j 2 \eta_{0}\left(k\left(C+(n-1) P_{r}\right)\right)+j 2 \eta_{0}(k C)}}{1-r_{n-1} r e^{-j 2 \eta_{0}\left(k\left(C+(n-1) P_{r}\right)\right)+j 2 \eta_{0}\left(k\left(C+(n-2) P_{r}\right)\right)}} .
\end{aligned}
$$




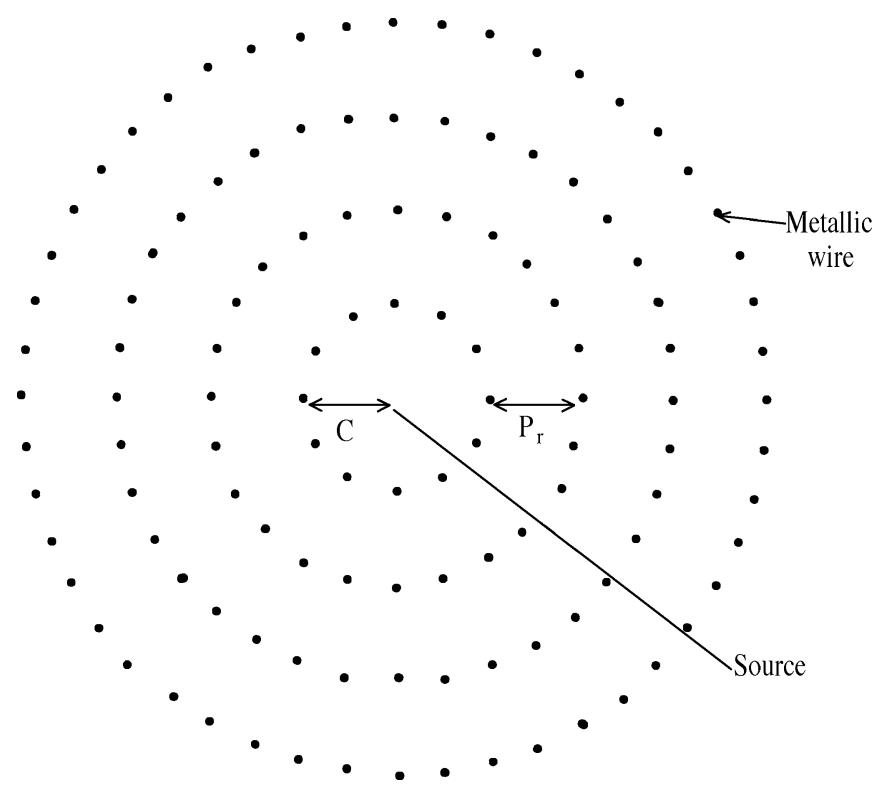

Fig. 7. CEBG structure composed of multiple layers of cylindrical FSSs.

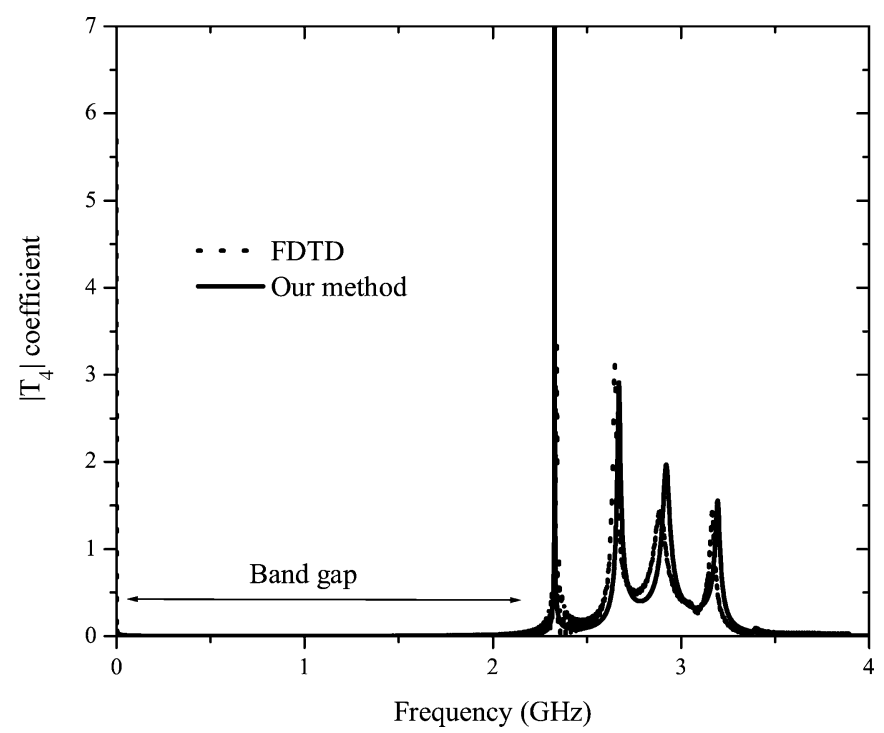

Fig. 8. Coefficient $\left|T_{n}\right|$ of a CEBG structure made of four layers $(n=4)$.

As an example, the coefficient $\left|T_{4}\right|$ of the structure shown in Fig. 7, with the parameters $C=P_{r}=45 \mathrm{~mm}, P_{\theta 1}=30^{\circ}$ and $a=1.5 \mathrm{~mm}$ is computed by the proposed approach and the FDTD method. Fig. 8 shows a comparison between both results. A good agreement is observed between the predicted results by our approach and those obtained using the FDTD method. The advantage of our proposed approach is that the computational time is considerably reduced compared to the FDTD method, which makes the design easier. From curves presented in Fig. 8, one can note that there is a first bandgap, where the value of $\left|T_{4}\right|$ is very low, and a passband, where the number of peaks is correlated with the number of cylindrical layers. The positions of the bandgap and the passband can be controlled by modifying the geometric parameters of the structure: $C, P_{t}, P_{r}$ and $a$. For the application presented in the next section, the end of the bandgap is positioned at $2 \mathrm{GHz}$.

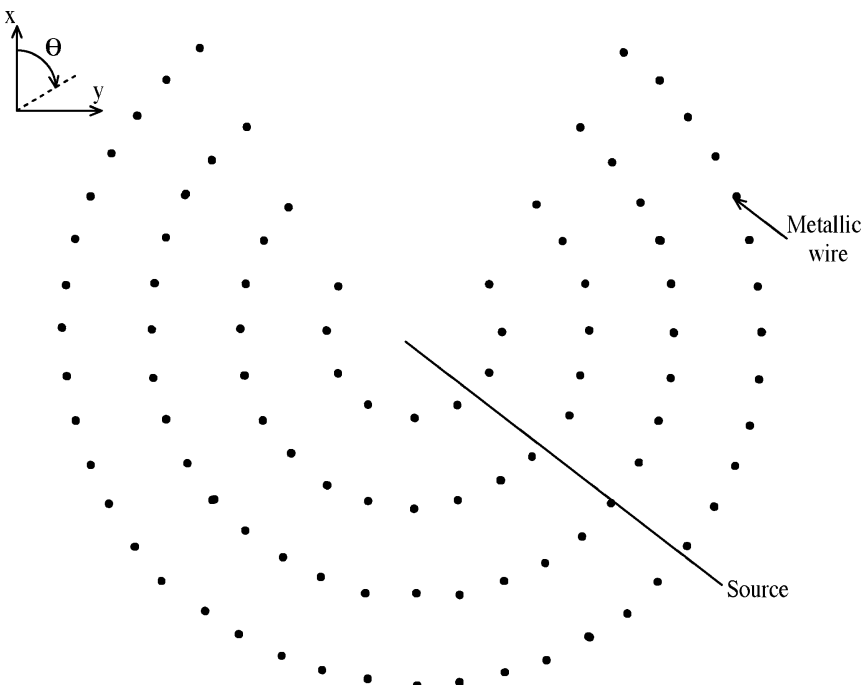

Fig. 9. Geometry of the CEBG structure with defects $\left(n=4, C=P_{r}=\right.$ $\left.45 \mathrm{~mm}, P_{\theta 1}=30^{\circ}\right)$.

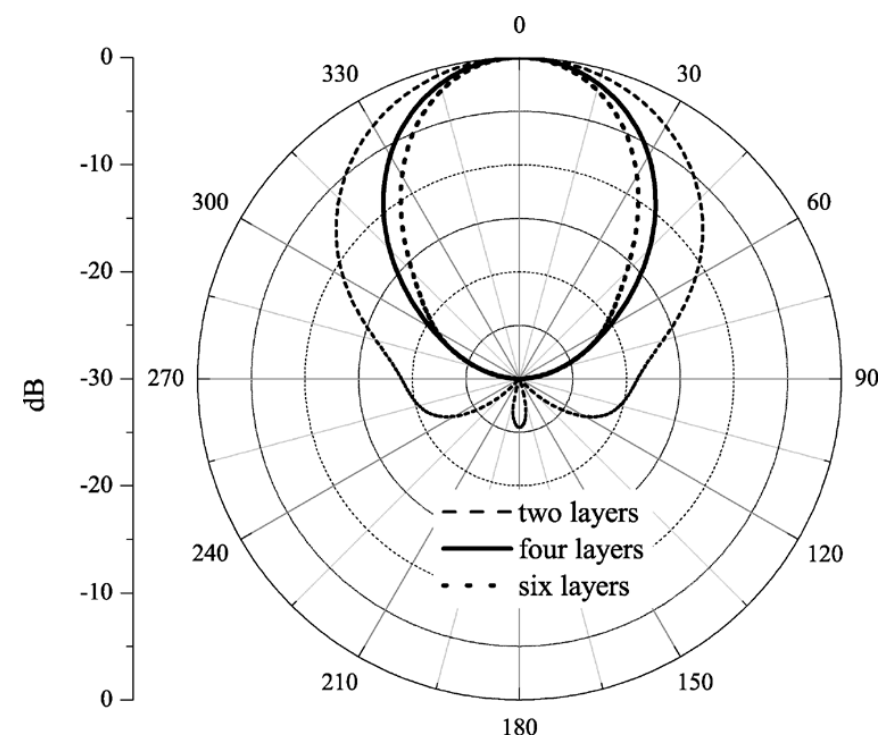

Fig. 10. Simulated radiation patterns in the $\mathrm{H}$-plane at $2 \mathrm{GHz}$ for different number of layers of the CEBG structure with defects consisting of removed wires.

\section{ApPlication to Reconfigurable DiRective Antennas}

The radiation pattern of CEBG structures, excited at their center, is omnidirectional as long as $P_{t} / \lambda<0.5$. To modify their radiation pattern, defects are introduced in the proposed structures. To demonstrate this approach, we consider the CEBG structure with defects, as illustrated in Fig. 9. In this example, defects consist of removing multiple wires: 3 wires are removed from the first layer, 5 from the second, 7 from the third, and so on.

For this case, numerical simulations using the FDTD method were carried out. Fig. 10 shows the radiation patterns in the H-plane of the CEBG structures with defects, for different number of layers, at $2 \mathrm{GHz}$ (this frequency is located at the end of the bandgap). From these curves, it can be seen that 


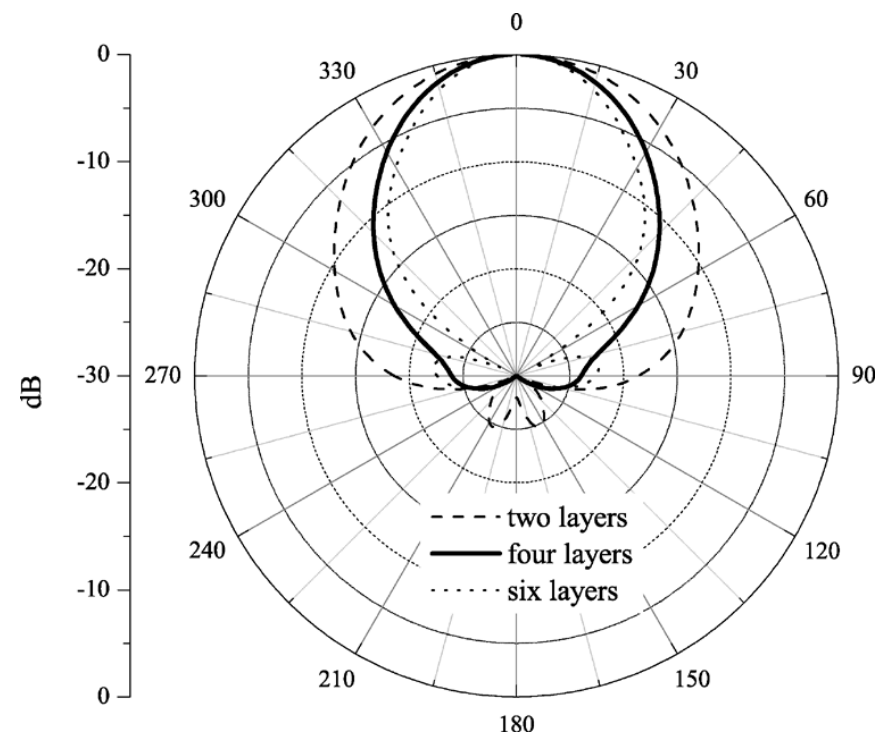

Fig. 11. Simulated radiation patterns in the $\mathrm{H}$-plane at $2 \mathrm{GHz}$ for different number of layers of the CEBG structure with defects consisting of discontinuous wires.

the directivity increases with the number of layers. The directivity is due to the electromagnetic wave modes opened by the defects in their direction and the electromagnetic waves forbidden in other directions caused by the existing bandgap. If we replace the continuous wires by discontinuous ones with diodes, where diodes are off for the removed wires and on for the remaining wires, we can note that the CEBG structure can allow an electronically sweeping of the antenna beam and reconfiguration of its radiation pattern over $360^{\circ}$ range. With this features it is possible to design reconfigurable antennas. These reconfigurable antennas have a potential application in mobile communications, and they can be used at the base station for reducing interferences and fading.

We consider now that the removed wires are replaced by discontinuous wires. The finite wires are $12 \mathrm{~mm}$ length and the vertical distance between two wires is $3 \mathrm{~mm}$. Fig. 11 shows the radiation patterns in the H-plane of the CEBG structures with defects consisting of discontinuous wires, for different number of layers, at $2 \mathrm{GHz}$. From this figure, it can be seen that the structures with discontinuous wires present the same desired radiation characteristic, i.e., a single directive beam, than those of the structures with removed wires.

As an example, an antenna made of a CEBG structure with defects (wires removed) is considered and studied in the next section.

\section{EXPERIMENTAL RESULTS}

A CEBG antenna prototype has been simulated with the FDTD method and fabricated to validate the proposed concept. The geometry of the CEBG illustrated in Fig. 9 is used. Fig. 12 shows a side view of this antenna. It consists of a monopole, as an excitation source, a ground plane, and a four-layer CEBG structure made of metallic wires (see Fig. 9).

In the FDTD simulations, the ground plane is considered infinite. The monopole has the same diameter as the parasitic wires,

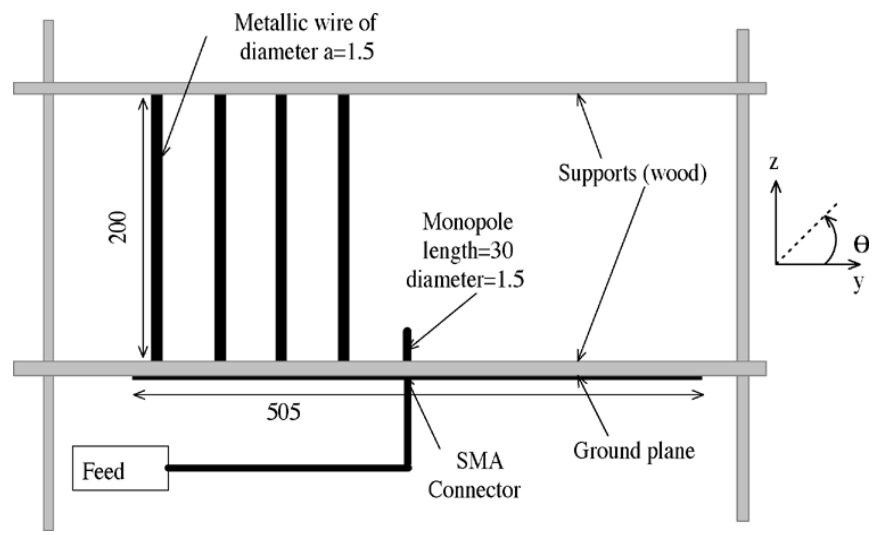

Fig. 12. Side view of the CEBG antenna (dimensions in millimeters).

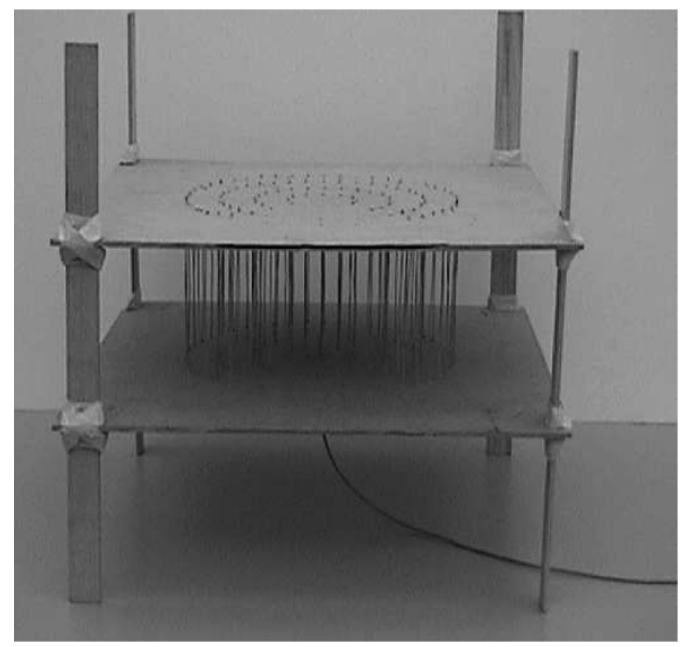

Fig. 13. Photograph of the fabricated CEBG antenna.

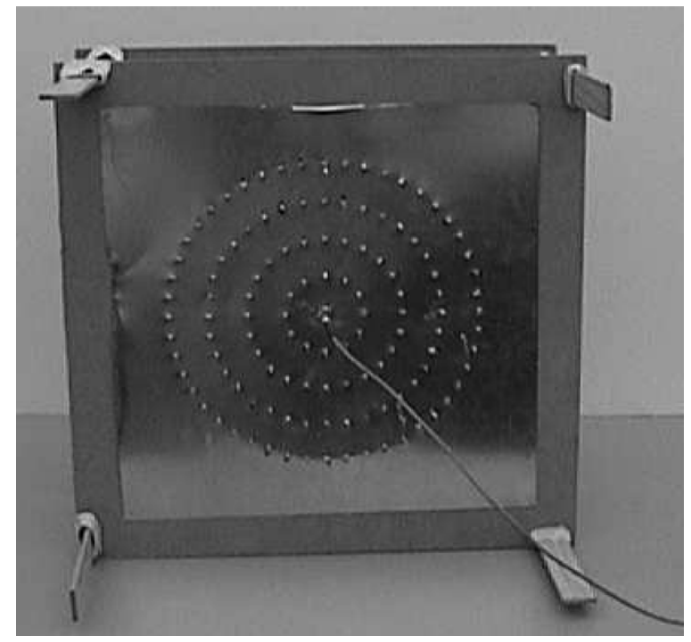

Fig. 14. Photograph of the fabricated CEBG antenna showing the connection and the ground plane.

and its length has been optimized to obtain matched impedance at $2 \mathrm{GHz}$. Figs. 13 and 14 show photographs of the fabricated CEBG antenna. The simulated and measured return loss of the antenna are shown in Fig. 15. A good agreement is obtained between theoretical and measured results. From the measured 


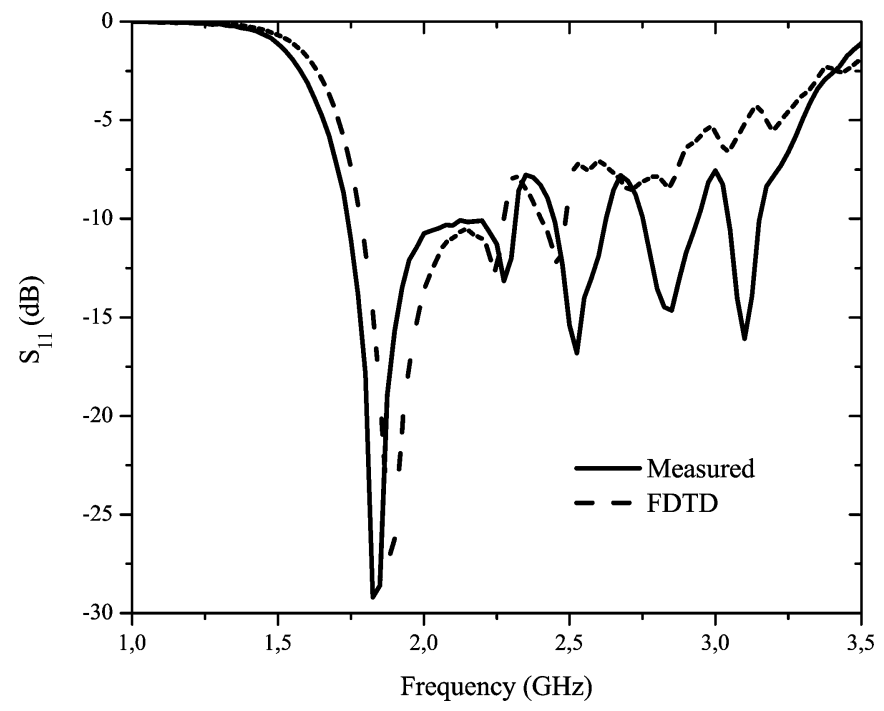

Fig. 15. Measured and simulated return loss of the proposed CEBG antenna.

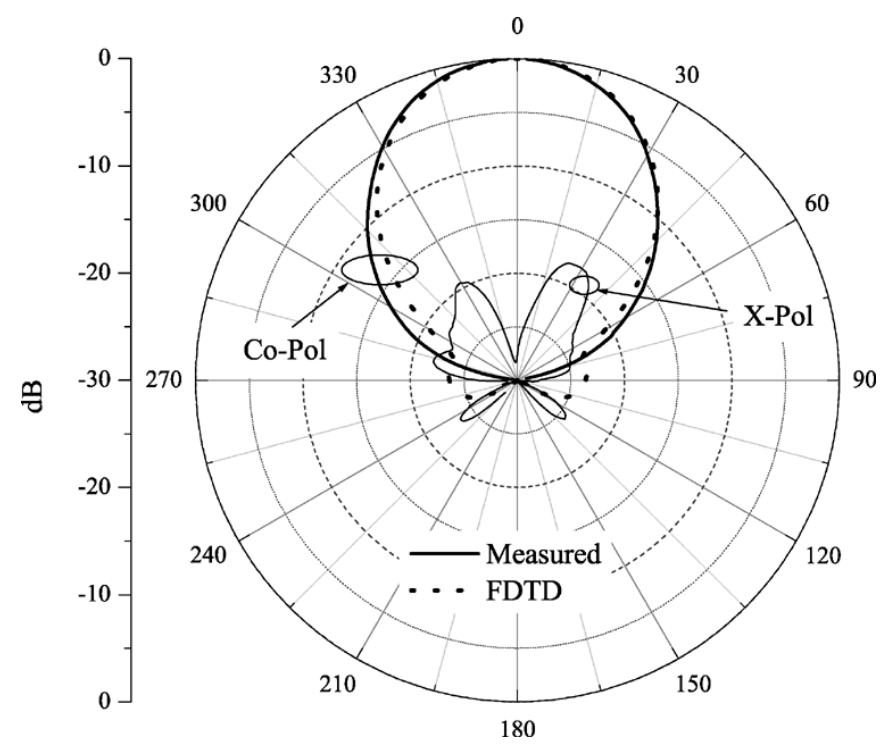

Fig. 16. Simulated and measured radiation patterns in the $\mathrm{H}$-plane at $1.77 \mathrm{GHz}$ (simulated X-pol is lower than $-30 \mathrm{~dB}$ ).

curve, a bandwidth $\left(S_{11}<-10 \mathrm{~dB}\right)$ from 1.74 to $2.31 \mathrm{GHz}$ (a fractional bandwidth of $28 \%$ ) is achieved, which is enough to cover the DCS, PCS, and UMTS bands (1.77-2.17 GHz). This CEBG antenna can find application for mobile communication base stations.

A further study of the CEBG antenna has focused on its radiation performance. The radiation patterns of the proposed antenna were measured in an anechoic chamber located at INRS, in Montreal. For comparison, numerical simulations for radiation patterns were also carried out using the FDTD method. The measured and simulated patterns are shown in Figs. 16-19. With reference to these curves, a good agreement between predictions and measured data can be observed. The half-power beam widths in the H-plane are $47.8^{\circ}$ and $37.9^{\circ}$ at 1.77 and $2.17 \mathrm{GHz}$, respectively. In the E-plane, the half-power beam widths are $36.3^{\circ}$ and $29.9^{\circ}$ at 1.77 and $2.17 \mathrm{GHz}$, respectively. The values of the maximum radiation in the cross polarization

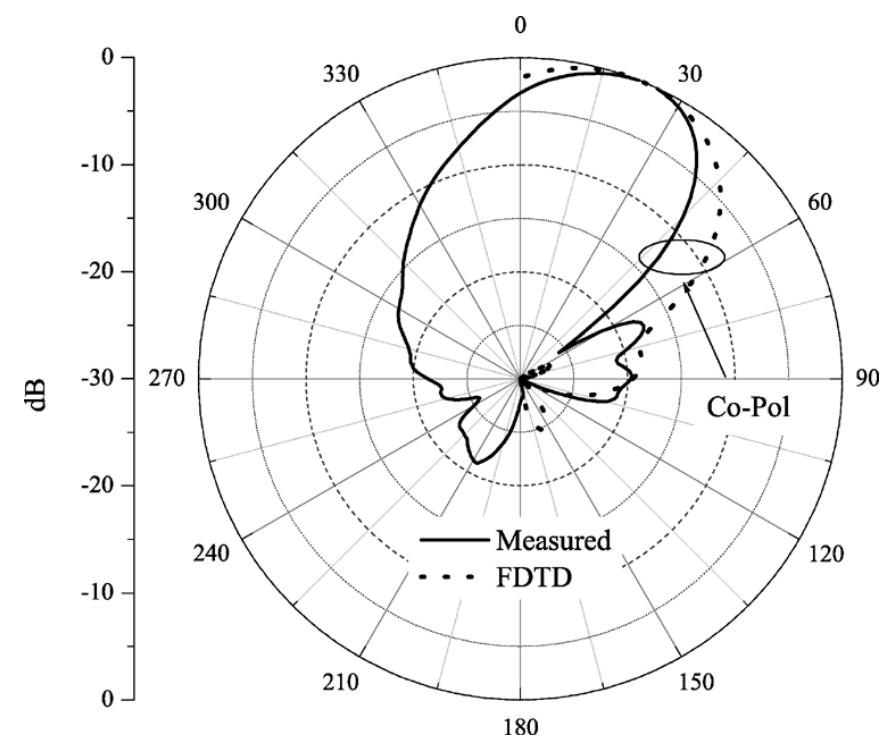

Fig. 17. Simulated and measured radiation patterns in the E-plane at $1.77 \mathrm{GHz}$ (simulated and measured X-pol are lower than $-30 \mathrm{~dB}$ ).

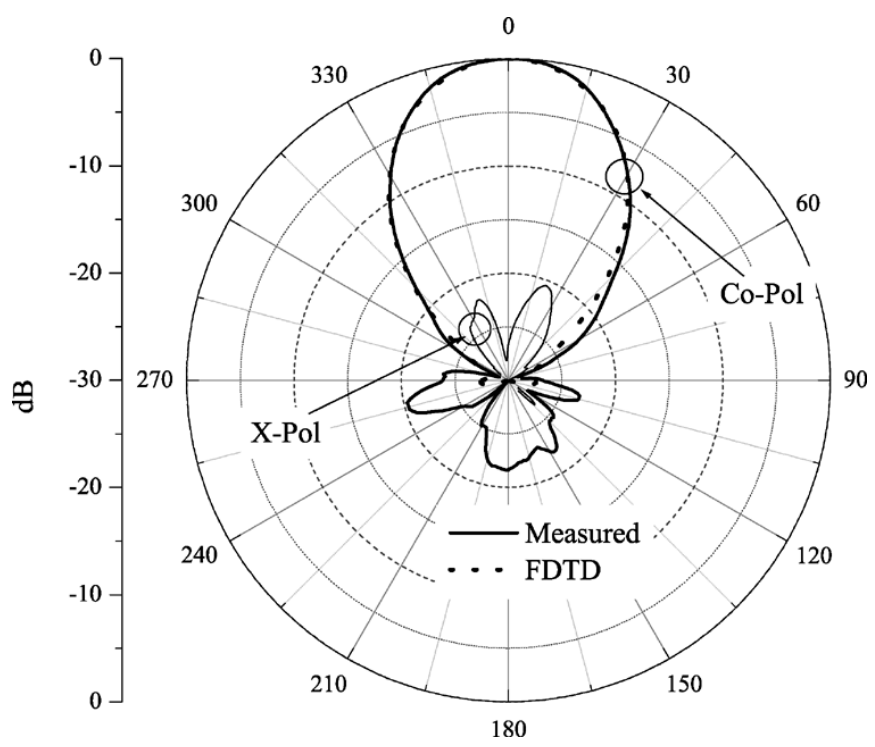

Fig. 18. Simulated and measured radiation patterns in the $\mathrm{H}$-plane at $2.17 \mathrm{GHz}$ (simulated X-pol is lower than $-30 \mathrm{~dB}$ ).

are $-18 \mathrm{~dB}$ and $-20.5 \mathrm{~dB}$ at 1.77 and $2.17 \mathrm{GHz}$, respectively. Note that in the E-plane the beam is slightly tilted. The angles of the tilt are $23.4^{\circ}$ at $1.77 \mathrm{GHz}$ and $19.8^{\circ}$ at $2.17 \mathrm{GHz}$. To explain the beam tilting, Fig. 20 presents the simulated radiation patterns in the E-plane of the CEBG antenna and of the monopole alone. From these curves, it can be seen that the beam tilting is essentially due to the monopole. Future work will concentrate on the examination of other sources that have a maximum radiation in the horizontal (e.g., a dipole antenna). The measured gains of the CEBG antenna are 12.2 and $15.8 \mathrm{~dB}$ at 1.77 and $2.17 \mathrm{GHz}$, respectively.

These measurements of CEBG antenna are unique and very positives. With such features, these antennas are suitable for wireless communication systems operating at $2 \mathrm{GHz}$, specially at the base station, where more gain is needed. 


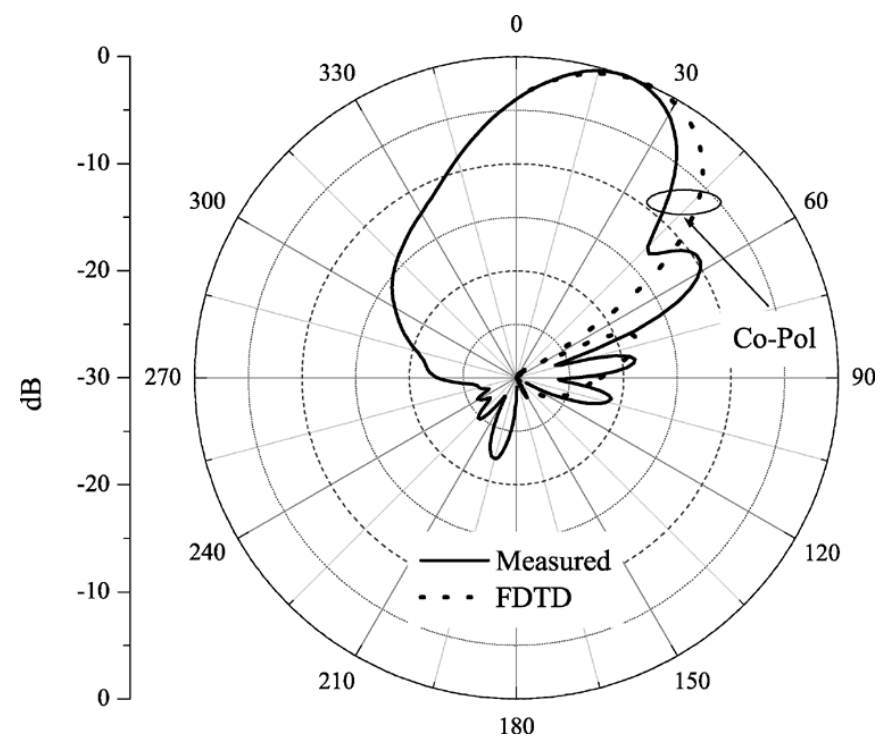

Fig. 19. Simulated and measured radiation patterns in the E-plane at $2.17 \mathrm{GHz}$ (simulated and measured X-pol are lower than $-30 \mathrm{~dB}$ ).

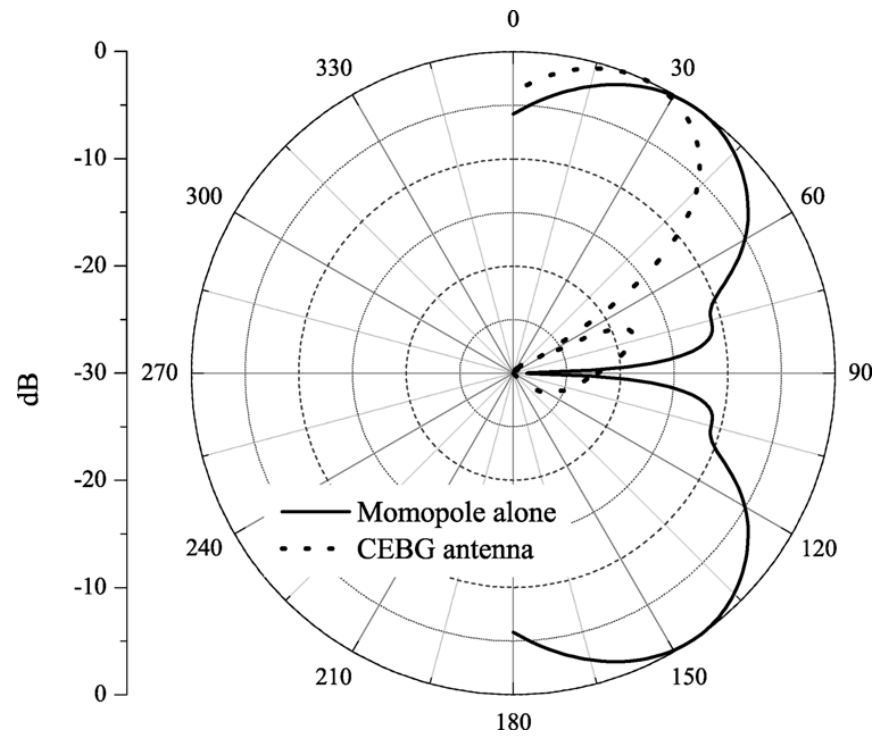

Fig. 20. Simulated Co-pol radiation patterns in the E-plane, at $2.17 \mathrm{GHz}$, of the monopole alone and the CEBG antenna.

\section{CONCLUSION}

This paper has presented the design of a new reconfigurable directive antenna by incorporating Cylindrical EBG structures composed of metallic wires. For this, a simple but efficient analysis of these periodic structures has been proposed. First, the transmission and reflection coefficients of a single cylindrical FSS have been extracted, and then, considering only fundamental mode interaction between layers, iterative relationships to handle multilayered Cylindrical EBG structures have been also developed and described. The obtained results by the proposed method have been successfully compared with that obtained using the FDTD method. Using the CEBG structures model, a prototype of a new directive antenna, without control, was designed, built and tested. This antenna offers a bandwidth of $28 \%$ and a gain between 12 and $16 \mathrm{~dB}$, which are enough for wireless applications, such as DCS, PCS, and UMTS.

The proposed CEBG antenna has several advantages, such as easy fabrication and a single feed, which can reduce complexity compared to feeding networks used in conventional antenna arrays. In addition, the gain can be increased by adding other layers and different types of exciting source can be used. Finally, due to the circular symmetry of the structure, it is possible to obtain a controllable radiation pattern over $360^{\circ}$ range by incorporating in the structure active components, such as diodes.

\section{REFERENCES}

[1] E. Yablonovitch, "Inhibited spontaneous emission in solid state physics," Phys. Rev. Lett., vol. 58, pp. 2059-2062, May 1987.

[2] J. Joannopoulos, R. D. Meade, and J. N. Winn, Photonic Crystals: Molding the Flow of Light. Princeton, NJ: Princeton Univ. Press, 1995.

[3] F. Yang and Y. Rahmat-Samii, "Microstrip antennas integrated with electromagnetic bandgap (EBG) structures: a low mutual coupling design for array applications," IEEE Trans. Antennas Propag., vol. 51, no. 10, pp. 2936-2946, Oct. 2003.

[4] M. Thevenot, C. Cheype, A. Reineix, and B. Jecko, "Directive photonic band-gap antennas," IEEE Trans. Microwave Theory Tech., vol. 47, no. 11, pp. 2115-2122, Nov. 1999.

[5] R. Biswas, E. Ozbay, B. Temelkuran, M. Bayandir, M. Sigalas, and K.-M. Ho, "Exceptionally directional sources with photonic band-gap crystals," J. Opt. Soc. Amer., vol. 18, pp. 1684-1689, Nov. 2001.

[6] J. M. Lourtioz, A. De Lustrac, F. Gadot, S. Rowson, A. Chelnokov, T. Brillat, A. Ammouche, J. Danglot, O. Vanbesien, and D. Lippens, "Toward controllable photonic crystals for centimeter and millimeter wave devices," J. Lightwave Tech., vol. 17, pp. 2025-2031, Nov. 1999.

[7] G. Poilasne, P. Pouliquen, K. Mahdjoubi, L. Desclos, and C. Terret, "Active metallic photonic bandgap material MPBG: experimental results on beam shaper," IEEE Trans. Antennas Propag., vol. 48, no. 1, pp. 117-119, Jan. 2000.

[8] R. C. Hall, R. Mittra, and K. M. Mitzner, "Analysis of multilayered periodic structures using generalized scattering matrix," IEEE Trans. Antennas Propag., vol. 36, no. 4, pp. 111-117, Apr. 1988.

[9] T. Cwik, "Coupling into and scattering from cylindrical structures covered periodically with metallic patches," IEEE Trans. Antennas Propag., vol. 32, no. 2, pp. 220-226, Feb. 1990.

[10] M. Mokhtar, "Analysis of cylindrical frequency selective surfaces," in Proc. Nat. Radio Science Conf. (NRSC98), Cairo, Egypt, 1998, pp. B81-B88.

[11] G. Loukos and J. C. Vardaxoglou, "Propagation inside strip grating FSS waveguides with cylindrical cross-section," Electron. Lett., vol. 31, pp. 778-779, May 1995.

[12] G. Gerini and L. Zappelli, "Multilayer array antennas with integrated frequency selective surfaces conformal to a circular cylindrical surface," IEEE Trans. Antennas Propag., vol. 53, no. 6, pp. 2020-2030, Jun. 2005.

[13] J. C. Eade, R. D. Seager, G. J. Cox, and J. C. Vardaxoglou, "A study of cylindrical resonant-structures constructed from frequency selective circuits," Int. J. Electron., vol. 88, pp. 695-705, Jun. 2001.

[14] H. Boutayeb, T. A. Denidni, A. Sebak, and L. Talbi, "Band structure analysis of crystals with discontinuous metallic wires," IEEE Microwave Wireless Comp. Lett., vol. 15, pp. 484-486, Jul. 2005.

[15] H. Boutayeb, K. Mahdjoubi, and A. C. Tarot, "Analysis of radius-periodic cylindrical structures," in Proc. IEEE AP-S Int. Symp. Dig., vol. 2, 2003, pp. 813-816.

[16] R. F. Harington, Time Harmonic Electromagnetic Fields. New York: McGraw-Hill, 1961

[17] H. Holland and L. Simpson, "Finite-diference analysis of EMP coupling to thin struts and wires," IEEE Trans. Electromagn. Compat., vol. 23, pp. 88-97, May 1981.

[18] R. E. Collin, Foundations for Microwave Engineering —Second Edition: McGraw-Hill International, 1992, ch. 5. 


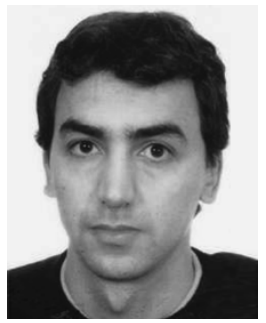

Halim Boutayeb (M'03) graduated in signal processing and telecommunications from the "Institut de Formation Supérieure en Informatique et Communication" (IFSIC) Rennes, France, in 2000 and received the D.E.A. degree (Master) in electronics in 2000 and the Ph.D. degree in signal processing and telecommunications from the University of Rennes, in 2003.

Since March 2004, he is a contracted Researcher with INRS-EMT, Montreal, Canada. His main fields of interest are electromagnetic bandgap structures applied to agile and directive antennas, FDTD method, and phased array antennas.

Dr. Boutayeb received the Best Paper Award at the JINA international conference in antenna in November 2004.

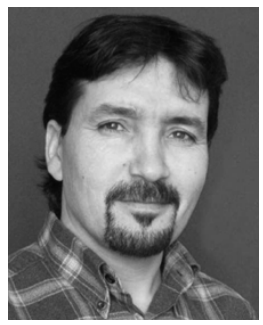

Tayeb A. Denidni (M'98-SM'04) received the B.Sc. degree in electronic engineering from the University of Setif, Setif, Algeria, in 1986, and the M.Sc. and Ph.D. degrees in electrical engineering from Laval University, Quebec City, QC, Canada, in 1990 and 1994, respectively.

From 1994 to 1996, he was an Assistant Professor with the engineering department, Université du Quebec in Rimouski (UQAR), Quebec, Canada. From 1996 to 2000, he was also an Associate Professor at UQAR, where he founded the Telecommunications laboratory. Since August 2000, he has been with the Personal Communications Staff, Institut National de la Recherche Scientifique (INRS-EMT), Université du Quebec, Montreal, Canada. He founded the RF laboratory, INRS-EMT, Monterial, for graduate student research in the design, fabrication, and measurement of antennas. He has 10 years of experience with antennas and microwave systems and is leading a large research group consisting of two research scientists, five Ph.D. students, and three M.S. students. Over the past ten years he has graduated many graduate students. He served as the Principal Investigator on many research projects on antennas for wireless communications. His current research interests include planar microstrip antennas, dielectric resonator antennas, EBG antennas, adaptive arrays, microwave and RF design for wireless applications, microwave filters, RF instrumentation and measurements, microwave and development for wireless communications systems. He has authored more than 40 papers in refereed journals. He has also had more than 80 papers and invited presentations in numerous national and international conferences and symposia.

Dr. Denidni is a member of the Order of Engineers of the Province of Quebec, Canada. He is also a member of the International Union of Radio Science (URSI) Commission C.

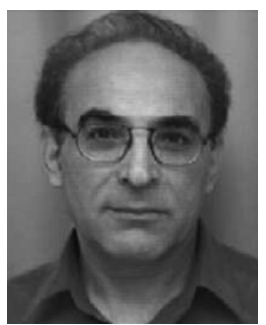

Kouroch Mahdjoubi (M'97) received the M.E.E. degree from the Engineering School of the Tehran University, Iran, in 1972, the D.E.A. (Master) diploma from ENSERG, Grenoble, France, in 1976, and the Doctor-Engineer and HDR diploma, from the University of Rennes 1, Rennes, France, in 1981 and 1998 , respectively.

In 1989, he joined the faculty of the Institute of Electronics and Telecommunications of Rennes (IETR), University of Rennes 1, where he is now a Full Professor. In 1997, he was a Visiting Professor with the Ecole Polytechnique, University of Nantes, France. He is with the Antennas and Microwaves Group, University of Rennes 1, and a former director of this Group. His areas of interest are electromagnetic modeling for antennas (FDTD, MoM), EBG antennas, metamaterials, and their antenna applications, antenna scattering, antenna arrays, and miniature antennas.

Dr. Mahdjoubi received the Best Paper Award at the JINA international conference in antenna in November 2004.

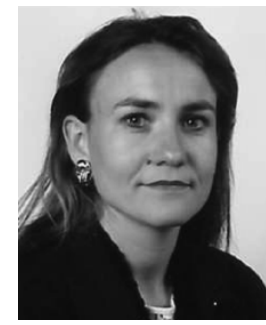

Anne-Claude Tarot (M'02) was born in 1969. She received the Ph.D. degree from the University of Rennes, Rennes, France in 1995.

Since 1998, she has been an Associate Professor at the Institute of Electronics and Telecommunications.of Rennes (IETR), University of Rennes. Her research interests include printed antennas, EBG materials and metamaterials.

Dr. Tarot received the Best Paper Award at the JINA Conference in Novvember 2004. She orgaJune 2005 (Saint-Malo, France) nized the 11th edition of ANTEM Symposium in

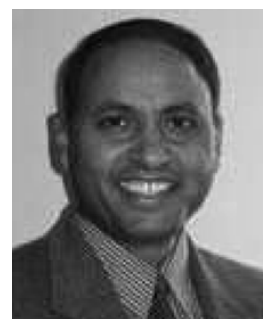

Abdel-Razik Sebak (M'81-M'84-SM'91) received the B.Sc. degree (with honors) in electrical engineering from Cairo University, Cairo, Egypt, in 1976, the B.Sc. degree in applied mathematics from Ein Shams University, Egypt, in 1978, and tthe M.Eng. and Ph.D. degrees from the University of Manitoba, Winnipeg, MB, Canada, in 1982 and 1984, respectively, both in electrical engineering.

From 1984 to 1986, he was with the Canadian Marconi Company, Kanata, Ontario, working on the design of microstrip phased array antennas. From 1987 to 2002, he was a Professor in the Electrical and Computer Engineering Department, University of Manitoba, Winnipeg. He is currently a Professor of Electrical and Computer Engineering, Concordia University, Montreal. His current research interests include phased array antennas, computational electromagnetics, integrated antennas, electromagnetic theory, interaction of EM waves with new materials and bio-electromagnetics.

Dr. Sebak is a member of the International Union of Radio Science Commission B. He received the 2003-2004 Faculty of Engineering, Concordia University double Merit Award for outstanding Teaching and Research. He has also received the 2000 and 1992 University of Manitoba Merit Award for outstanding Teaching and Research, the $1994 \mathrm{Rh}$ Award for Outstanding Contributions to Scholarship and Research in the Applied Sciences category, and the 1996 Faculty of Engineering Superior Academic Performance. He has served as Chair for the IEEE Canada Awards and Recognition Committee (2002-2004) and IEEE Canada CONAC (1999-2001). He has also served as Chair of the IEEE Winnipeg Section (1996-97). He served as the Treasurer (1992, 1996, and 2000) and Publicity Chair (1994) for the Symposium on Antenna Technology and Applied Electromagnetics (ANTEM). He has also served as Chair (1991-92) of the joint IEEE AP/MTT/VT Winnipeg Chapter. He received, as Chapter Chair, the 1992 IEEE Antennas and Propagation Society Best Chapter Award.

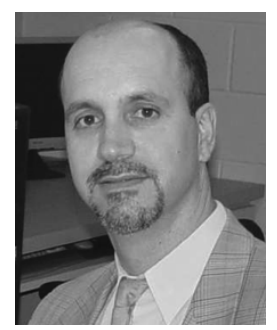

Larbi Talbi (S'95-A'97-SM'05) received the M.S. and Ph.D. degrees in electrical engineering from Laval University, Quebec, Canada, in 1989 and 1994.

He completed a Postdoctoral Fellowship at INRS-Telecommunications, Montreal, QC, Canada, within the Personal Communications Systems Group between 1994 and 1995, where he led two projects supported by Bell-Canada. From 1995 to 1998, he was an Assistant Professor in the Electronics Engineering Department at Riyadh College of Technology, Saudi Arabia. From 1998 to 1999, he was with the Electrical and Computer Engineering Department of Laval University, as an invited Professor. He is currently a Professor at the Department of Computer Science and Engineering at the University of Quebec in Outaouais, Gatineau, Canada, and also as an Associate Professor at INRS-EMT, Montreal, Canada. Since August 2005, he has been with the Propagation and Satellite (PSAT) Labs of Communications Research Center(CRC ), Ottawa, Canada, for a sabbatical leave. His research activities include experimental characterization and modeling of UHF/EHF indoor radio propagation channels and anti-multipath techniques for indoor wireless LAN, design of MIMO integrated antennas and microwave circuits for wireless communication systems, Dielectric properties measurement techniques, and radar cross sections.

Dr. Talbi is a member of the Ordre des ingénieurs du Québec, and of numerous Technical Program Committees. 